\title{
Establishment and application of information resource of mutant mice in RIKEN BioResource Research Center
}

\author{
Hiroshi Masuya1(D, Daiki Usuda', Hatsumi Nakata², Naomi Yuhara', Keiko Kurihara', Yuri Namiki', Shigeru Iwase', \\ Toyoyuki Takada', Nobuhiko Tanaka', Kenta Suzuki ${ }^{1}$, Yuki Yamagata ${ }^{3}$, Norio Kobayashi ${ }^{1,4}$, Atsushi Yoshiki ${ }^{2}$ and \\ Tatsuya Kushida'
}

\begin{abstract}
Online databases are crucial infrastructures to facilitate the wide effective and efficient use of mouse mutant resources in life sciences. The number and types of mouse resources have been rapidly growing due to the development of genetic modification technology with associated information of genomic sequence and phenotypes. Therefore, data integration technologies to improve the findability, accessibility, interoperability, and reusability of mouse strain data becomes essential for mouse strain repositories. In 2020, the RIKEN BioResource Research Center released an integrated database of bioresources including, experimental mouse strains, Arabidopsis thaliana as a laboratory plant, cell lines, microorganisms, and genetic materials using Resource Description Framework-related technologies. The integrated database shows multiple advanced features for the dissemination of bioresource information. The current version of our online catalog of mouse strains which functions as a part of the integrated database of bioresources is available from search bars on the page of the Center (https://brc.riken.jp) and the Experimental Animal Division (https://mus.brc.riken.jp/) websites. The BioResource Research Center also released a genomic variation database of mouse strains established in Japan and Western Europe, MoG ${ }^{+}$(https:// molossinus.brc.riken.jp/mogplus/), and a database for phenotype-phenotype associations across the mouse phenome using data from the International Mouse Phenotyping Platform. In this review, we describe features of current version of databases related to mouse strain resources in RIKEN BioResource Research Center and discuss future views.
\end{abstract}

Keywords: Bioresource, Mouse mutation information resource, Database, data integration, Semantic web, Ontology

\section{Introduction}

Mouse is one of the most important model organisms for studying biological phenomena in mammals. In particular, mice are prominent in human biology and disease research due to their genetic, genomic, phenotypic, and physiologic proximity to human. In the mammalian genetics research community, a number of genetically

\footnotetext{
* Correspondence: hiroshi.masuya@riken.jp

${ }^{1}$ Integrated Bioresource Information Division, RIKEN BioResource Research Center, 3-1-1 Koyadai, Tsukuba-shi, Ibaraki 305-0074, Japan

Full list of author information is available at the end of the article
}

modified (GM) mice have been established using various artificial genetic engineering technologies such as transgenic, gene-targeting, and genome editing which are useful as in vivo models of human disease. Sharing of same mutant mouse strains across studies is essential to ensure reproducibility of experimental data. Online databases serve as an information resource for mouse mutant strains and play crucial a role providing: 1) a catalog of significant unique resources; and 2) a portal of genetic/phenotypic characteristics of the strains which is useful when designing experimental plans. In

C C The Author(s). 2021 Open Access This article is licensed under a Creative Commons Attribution 4.0 International License, which permits use, sharing, adaptation, distribution and reproduction in any medium or format, as long as you give appropriate credit to the original author(s) and the source, provide a link to the Creative Commons licence, and indicate if changes were made. The images or other third party material in this article are included in the article's Creative Commons licence, unless indicated otherwise in a credit line to the material. If material is not included in the article's Creative Commons licence and your intended use is not permitted by statutory regulation or exceeds the permitted use, you will need to obtain permission directly from the copyright holder. To view a copy of this licence, visit http://creativecommons.org/licenses/by/4.0/ The Creative Commons Public Domain Dedication waiver (http://creativecommons.org/publicdomain/zero/1.0/) applies to the data made available in this article, unless otherwise stated in a credit line to the data. 
addition, global data sharing using data integration technology is key.

Modern biology has been accelerated by global sharing of biological data. The global community of mouse biology and genetics historically evolved via the accumulation of knowledge of mutant strains that helps transfer of knowledge among studies, hypothesis building, and experimental reproducibility by use of common mouse resources as experimental material which carries equivalent genetic characteristics. For example, Mouse Genome Informatics (MGI) greatly helps rapid accumulation of genetics/genomics knowledge [1, 2]. In cooperation with nomenclature standards for mouse genes and strains, MGI gives unique identifiers (UIDs) to data items in the database with name space of "MGI" such as "MGI:xxxxx" which is commonly used in multiple public databases in life science [3]. The description of genetic markers and mouse strains using a common identifier helps data access and integration and improves interoperability and reusability of data across databases. In mammalian research, authoritative data are provided by the MGI, the
HUGO Gene Nomenclature Committee (HGNC), and the Rat Genome Database (RGD) with nomenclature activities for genes, alleles, and strains for each species [3-5]. Data from the National Center for Biotechnology Information (NCBI: https://www.ncbi.nlm.nih.gov) and Ensembl (https://ensembl.org) are also broadly used across species to provide identifiers [6, 7]. The International Mouse Strain Resource (IMSR), a combined catalog of worldwide mouse resources with direct access to repository sites holding resources [8], also records UIDs of mouse strains given by original repositories.

In the last 20 years, UID-based data sharing and integration have been performed on various types of data across databases. The Open Biomedical Ontology (OBO) Consortium provides sets of controlled vocabularies termed "ontologies" for complicated concepts such as gene function managed by UIDs (e.g. "GO:0000016" for "lactase activity") developed with the aim of comprehensive annotation of biological information $[9,10]$.

In the MGI, ontologies for anatomical body parts (Mouse Anatomy Ontology: MA) [11] and phenotype

\section{A Basic expression of RDF data model (a triple)}

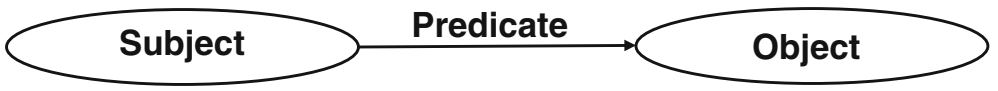

\section{B Example of description for a mouse mutant}

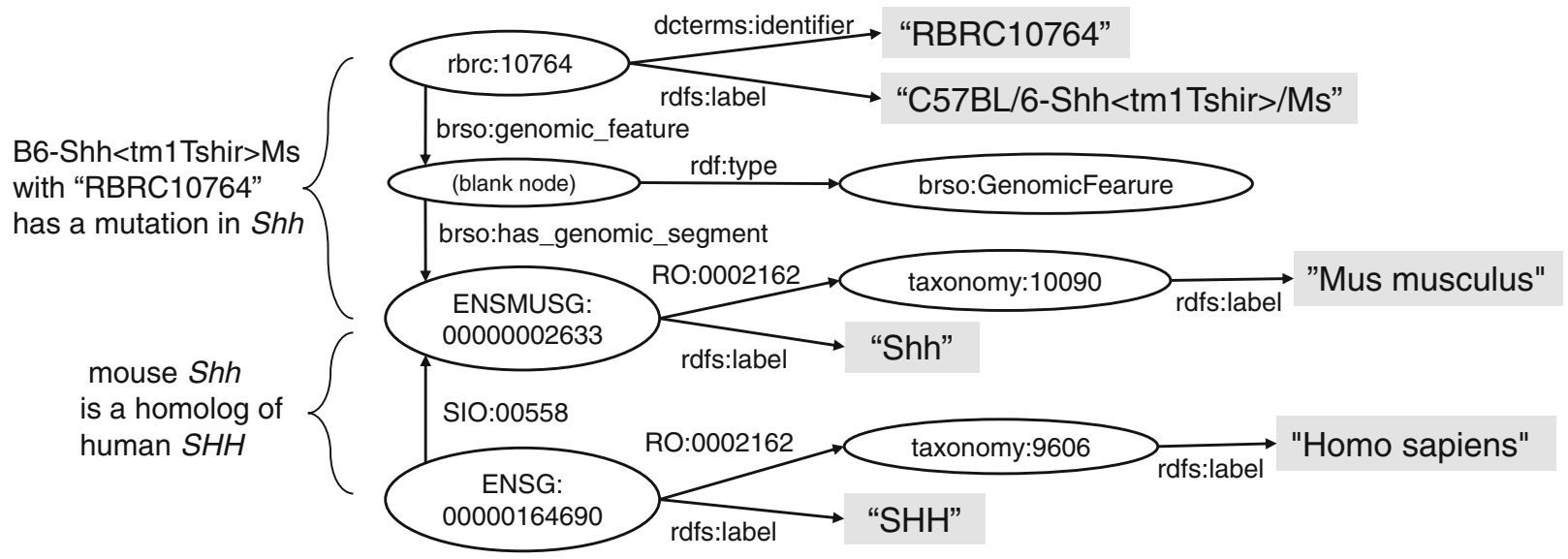

\section{Abbreviations (prefixes)}

rbrc: http://metadb.riken.jp/db/rikenbrc_mouse/ brso: http://purl.jp/bio/10/brso

rdf: http://www.w3.org/1999/02/22-rdf-syntax-ns\# rdfs: http://www.w3.org/2000/01/rdf-schema\#

\author{
dcterms: http://purl.org/dc/terms/ \\ ENSMUSG: http://rdf.ebi.ac.uk/resource/ensembl/ENSMUSG \\ ENSG: http://rdf.ebi.ac.uk/resource/ensembl/ENSG \\ taxonomy: http://identifiers.org/taxonomy/ \\ RO: http://purl.obolibrary.org/obo/RO \\ SIO: http://semanticscience.org/resource/SIO_
}

Fig. 1 Basic semantic expression of RDF data model. a RDF data model is based on the simple expression in a "triple" which is a set of three entities, subject, predicate and object to describe resources in the WWW. Subjects and predicates are addressed in URIs. An object is addressed in an URI or text. $\mathbf{b}$ An example of RDF data model forming a knowledge graph describing a mouse mutant represented in multiple triples. Each URIs are represented with abbreviations (prefixes) listed in the box at the bottom part. Note that most URIs were defined outside RIKEN (as common vocabularies). Upper parts of the graph describe rbrc:10764 named as "C57BL/6-Shh < tm1Tshir>/Ms" with identifier "RBRC10764" has a mutation in the Shh gene of Mus musculus. The blank node is an anonymous record representing a genomic feature of C57BL/6-Shh < tm1Tshir $>$ / Ms. (rbrc:10764). Lower part describes Shh is a homolog of the SHH gene of Homo sapiens 
(Mammalian Phenotype Ontology: MP) [12] were developed for annotation of characteristics of mutant strains. Using these ontologies, completeness of searching has been significantly improved in MGI and RGD $[12,13]$. Ontology also helps representing meaning of terms or concepts by its graph structure composed of links among UIDs. The development of methodologies for computational comparison between phenotype ontology terms allows linking of phenotype records of model animals to diseases which are annotated with Human Phenotype Ontology (HPO) automatically [14-19]. Therefore, description of mouse strains themselves and their metadata (e.g. genetic and phenotypic features) using UID-based data items such as ontologies is remarkably beneficial for strain repositories to represent how mouse strains can be useful for disease studies. In addition, Research Resource Identifiers (RRIDs) are proposed to help researchers citing bioresources used in their study [20] including, antibodies, model organisms, and tools (i.e. software and databases).

There is a growing concern regarding the capacity of computational systems as a result of the increase in volume, complexity, and creation speed of data. The "semantic web" offers a series of methods and technologies to develop extensions of the current World Wide Web (WWW) in which information is given well-defined meanings and integration [21]. These technologies include the Resource Description Framework (RDF) [22] which is a framework to provide a formal description of concepts, terms, and relationships within a given knowledge of domains recommended by the World Wide Web Consortium (W3C). In the RDF, informational resources on the Web are named by unique resource identifiers (URIs) which work as UID on the WWW. URIs in RDF are usually described as uniform resource locators (URL) on the Web. URL helps to show where you need to access to obtain more information about the resource. The expressiveness allows RDF to define controlled vocabularies with exact relationships to other informational resources. RDF represents a powerful data model for data federation and interoperability across various datasets. The data model of RDF, which forms knowledge graphs composed of interlinking URIs, provides interoperability between applications that exchange machine-readable information on the Web (Fig. 1). Currently, multiple database management systems that store and process RDF data (often termed as "triples" because RDF data model is described in expression of the subject-predicateobject: see Fig. 1a) are called RDF stores or triple stores as introduced in https://db-engines.com/en/article/RDF+Stores. The common query language of RDF, SPARQL, ${ }^{1}$ which allows for a query to consist of triple patterns, conjunctions, disjunctions, and optional patterns is openly provided and can act as a common Application Programing Interface $(\mathrm{API})^{2}$ of RDF stores on the Web. Furthermore, the "federated query" extension of SPARQL ${ }^{3}$ allows executing queries distributed over different SPARQL endpoints. Using this extension, users can easily merge data distributed across the Web. From these features, RDFbased data integration is considered to be one of the basic strategies for making research results available through the Web.

In 2016, FORCE11, a community of scholars, librarians, archivists, publishers, and research funders that has arisen organically to help facilitate the change toward improved knowledge creation and sharing formulated FAIR Data Principles (https://www.force11.org/group/ fairgroup/fairprinciples) ${ }^{4}$ act as foundational guidelines to improve the findability, accessibility, interoperability, and reuse of digital assets. It is suggested that there is an urgent need to improve the infrastructure that supports the reuse of academic data according to the FAIR principle [23]. Hence, data integration technology becomes non-negligible for repositories of bioresource due to efficient use of resources in the scientific community.

The RIKEN BioResource Research Center (BRC), a global not-for-profit public institution providing biological materials, technical services, and educational programs, has collected, preserved, and provided experimental mice, Arabidopsis thaliana as a laboratory

\footnotetext{
${ }^{1}$ SPARQLSPARQL (a recursive acronym for "SPARQL Protocol and RDF Query Language") is a query language for retrieval and manipulation of data stored in RDF format. SPARQL has been standardized by the RDF Data Access Working Group (DAWG) in WWWC

${ }^{2}$ APIApplication Programming Interface (API) is a computing interface used by software components to exchange information with each other. The important role of the API is to provide programmatic operation method (interface) for a software application from the other software applications. APIs allow developers to manipulate remote resources through protocols, specific standards for communication that allow different technologies to work together.

${ }^{3}$ Federated query extension of SPARQLFederated search retrieves information from multiple data sources through a search engine by the process that single query request is distributed to multiple databases. In SPARQL, federated query protocol allows to build a query to be distributed to multiple SPARQL endpoints (services that accept SPAR QL queries and return results) and results gathered.

${ }^{4}$ FAIR data principlesThe FAIR Data Principles are proposed by a voluntary group of researchers, librarians and publishers called FORCE 11 (Future of Research Communications and e-Scholarship) aiming creation and sharing of knowledge in 2014. The Principles provides a set of guiding principles making data findable, accessible, interoperable and reusable to provide guidance for scientific data management and stewardship to promote maximum use of research data. Capacity of computational systems to find, access, interoperate, and reuse data with minimal human intervention are extremely important elements for the Principles because humans increasingly rely on computational support to deal with huge data. Practical hints on how they could be implemented is explained at http://fairprinciples.org/.
} 
plant, cell lines derived from humans and animals, microorganisms, and associated genetic materials. Mice are one of the major resources in RIKEN BRC. In 2019, RIKEN BRC collected over 8800 mouse strains including inbred, spontaneous mutant, recombinant inbred, consomic, transgenic, knockout, wild-derived, and genome editing [24]. RIKEN BRC develops its informatics activity to ensure the wide dissemination of bioresource data and contributes to develop novel utilities and create new "values" and facilitate wide, effective and efficient use of bioresources for R\&D in science and industry. The core activities of RIKEN BRC's informatics include: 1) data integration and standardization aiming wider dissemination of bioresource data and additional metadata for biological properties of bioresources such as genome, phenotype, and conceivable research use in collaboration with other biological databases and medical data; 2) improvement of homepage contents to provide userfriendly interface for biologists; and 3) big data analysis aiming to facilitate novel utilities with discoveries of hidden values of bioresources. Here, we review the development of mouse strain resources in RIKEN BRC as the basic infrastructure for promotion of the life science.

\section{Main text \\ Development of an information resource of mutant mice using data integration technologies in RIKEN BRC Bioresource online catalog}

Aiming to meet the needs summarized in the FAIR principles toward the wider dissemination of bioresource information and thereby adding value to bioresources, RIKEN BRC has been applying data integration technologies based on the RDF-related technologies for database of bioresources [25, 26]. There are number of advantages when using RDF for bioresource database. Firstly, the RDF data model forming graphs (instead of tables in relational database) is flexible. New data is easily added by adding a new column and updating the schema. This feature is suitable for bioresource databases to add a variety of information or data of biological characteristics to bioresources. Since bioresource usage is greatly influenced by scientific trends, it is extremely important that they can be easily expanded according to the needs. Secondly, it is easy to integrate bioresource information with other RDF-based public datasets in life science. In the bioresource database, description of genetic and phenotypic characteristics is essential to promote the use of bioresources. RDF provides a simple way of description of biological characteristics referring URIs of public data of genetic markers which involves gene and alleles and ontology terms of phenotypic characteristics bioresources. Thirdly, RDF provides fundamental infrastructure of data dissemination on the Web. If the RDF-based statement of bioresources is openly available on the Web, anyone can refer an URI of a bioresource for description of an experimental result, allowing the establishment of the exact relationship of the bioresource and the experiment on the Web. This helps to ensure reproducibility of experimental data.

The current version of online catalog of mouse strains in RIKEN BRC is implemented as part of the integrated database of whole five bioresources in RIKEN BRC including, experimental mice, Arabidopsis thaliana, cell lines, microorganisms, and genetic materials. The database is composed of a back-end database engine called "RDF store" and a front-end software program to generate dynamic Web-based GUI for retrieval to RDF store showing search results of mouse strains (Fig. 2).

In the back-end RDF store, implemented with Virtuoso (OpenLink Software Inc.), basic data of bioresources in RIKEN BRC including mouse strains, mouse gene and allele data imported from MGI [2] and RDF version of Ensembl (https://www.ebi.ac.uk/rdf/documentation/ ensembl/) [27] are stored. In the RIKEN BRC, mouse strains are managed with UIDs composed of "RBRC" (meaning RIKEN BRC) prefix and strain number. For the generation of RDF versions of mouse strain data, the URI of mouse strain records are generated referring RBRC-number. Basic data of mouse strains contains strain's UID, name, synonym(s) for name, back ground strain, description for strain development and phenotype, link to health report, gene mutation (allele), specific term and conditions for use of the strain, status of preservation, and publication among others. Genes and alleles mutant strains have been described with common UIDs and URIs. Data imported from MGI and Ensembl are used to allow searching by gene name, synonyms, and name of homologs.

The Web interface of mouse strain search is integrated in the RIKEN BRC (https://brc.riken.jp) and the Experimental Animal Division (https://mus.brc.riken.jp/ ) websites (top-page of the both websites). Suggestions of gene and strain names are listed according to the characters entered by the user dynamically. The search result is represented as a summarized list of strains that meet the search condition. The detailed information page of a mouse strain is linked from the summarized list page (Fig. 3).

In addition to the mouse strain search interface, users can directly access to mouse strain data via another Web interface (https://knowledge.brc.riken.jp/bioresource/db/ xsearch_animal) that is a direct interface of the back-end RDF store operated by "Bioresource MetaDatabase" (https://knowledge.brc.riken.jp/) which is an instance of the RIKEN MetaDatabase software application [26]. The Bioresource MetaDatabase provides a simple common view of RDF, download function of RDF data, and a 


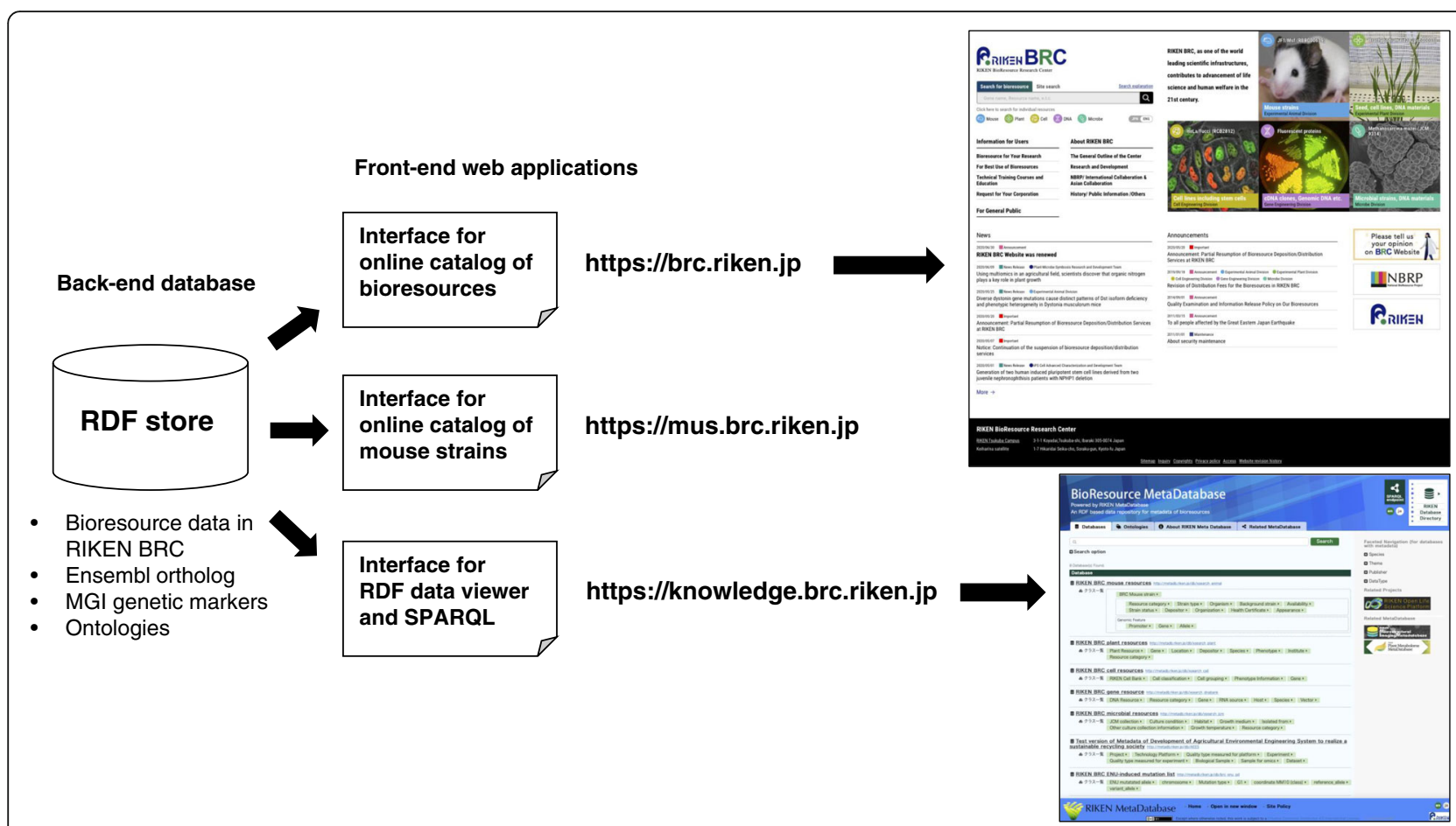

Fig. 2 Overview of bioresource integrated database in RIKEN BRC. Left panel shows composition of the bioresource integrated database. The integrated database for online catalog of bioresources in RIKEN BRC is composed of a back-end database and the front-end web applications. Three different applications (online catalogs of whole bioresources in RIKEN BRC, mouse and RDF data viewer and SPARQL search) are hosted by the back-end database. Right panel shows web interfaces of https://brc.riken.jp and https://knowledge.brc.riken.jp. Interface of https://mus.brc. riken.jp is shown in Fig. 2

SPARQL endpoint that accepts SPARQL queries and returns results. Using the SPARQL query language, users can output data in a user-specified format from the endpoint. In particular, using federated query syntax of SPARQL, users can obtain combined results between mouse strains and related biological information such as genome, proteins, and diseases which are hosted in other SPARQL endpoints such as the EBI RDF platform (https://www.ebi.ac.uk/rdf/) [27] and DisGeNET (https:// www.disgenet.org) [28]. Mouse strain data in RIKEN BRC is regularly uploaded and available at IMSR which is an international portal of mouse strain repositories (http:// www.findmice.org) [8]. Assignment of RRID for mouse strains in RIKEN BRC is already done via the IMSR database (e.g. RRID: IMSR_RBRC09538).

\section{Genetic variation database "MoG"}

Studies of genetic and genomic variation are essential to understand the genetic contribution and pathogenesis of diseases in human. As a mammalian model of genetic variation of human, genetically diverse mouse inbred strains provide multiple important biological insights [29]. To provide mouse genetic variation compared with inbred mouse strains established in Japan (JF1/Ms. etc), and frequently used classical inbred strain such as
C57BL/6 J (whose genome is predominantly derived from the West European subspecies M. m. domesticus), RIKEN BRC operates a mouse genetic variation database named "MoG" (pronounced móg $\mathrm{pl}^{+} \mathrm{s}$ ) which is an expanded version of a genome database, NIG_MoG, transferred from the National Institute of Genetics [30, 31] (Fig. 4). $\mathrm{MoG}^{+}$takes over polymorphism information on nucleotide substitutions and short indels, focusing on the protein-coding genes of the two M. m. molossinusderived strains with respect to those of $\mathrm{B} 6$ and other representative classical inbred mouse strains based on the results of re-sequencing experiments. The GUI of $\mathrm{MoG}^{+}$provides gene search, BAC clones deposited in RIKEN BRC, and genomic region. Search results are visualized as a map view, sequence alignment, details of coding region and gene feature, and detailed information of BAC.

\section{Contribution to the international mouse Phenotyping Consortium and provision of an advanced reference data set}

The International Mouse Phenotyping Consortium (IMPC) was established in 2011 [32] expanding international collaborative networks in phenotyping knockout mice, with the goal to publish an Encyclopedia of the Mammalian 


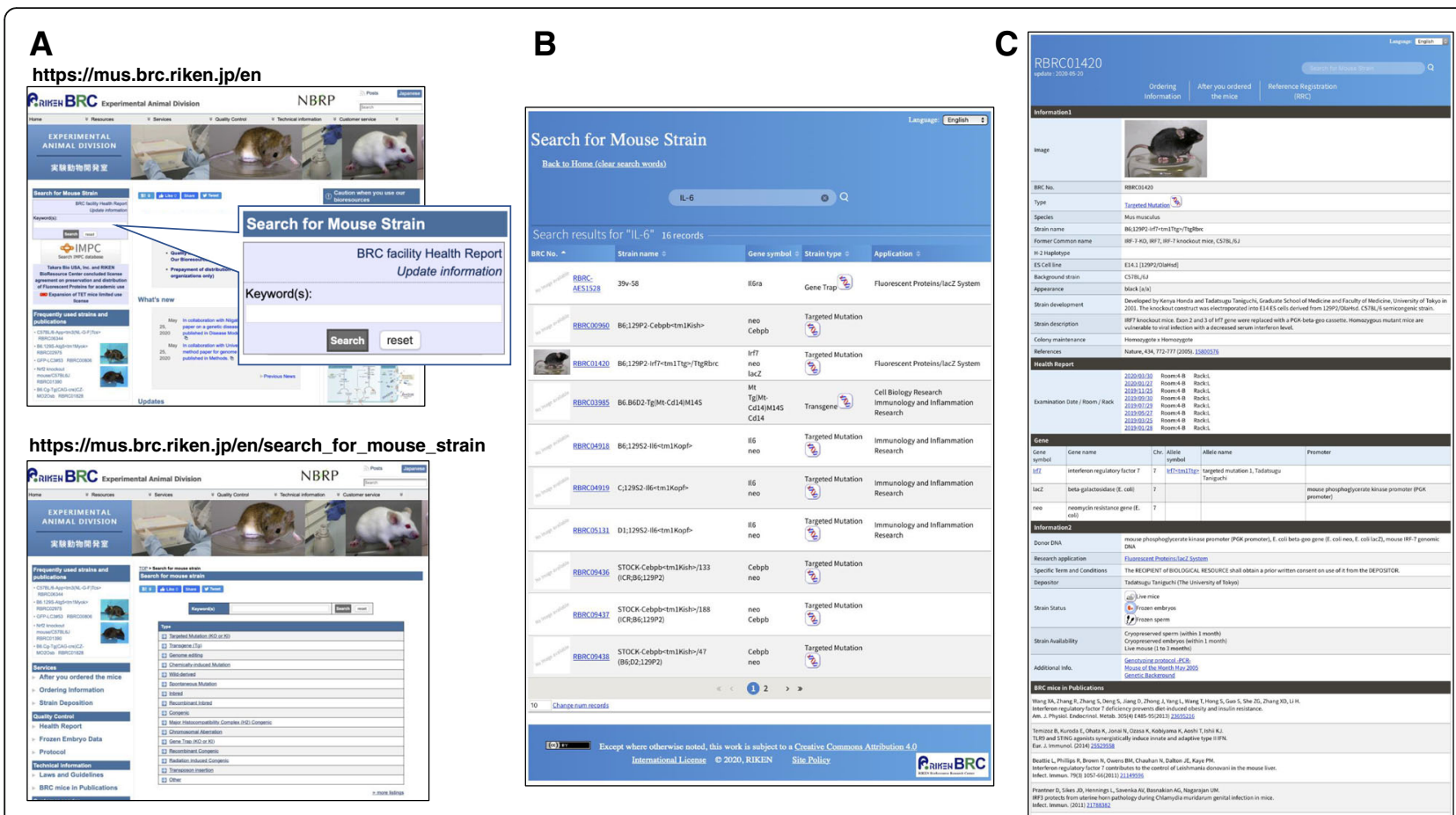

Fig. 3 Overview of mouse strain search. a Search bars of mouse strain in RIKEN BRC are represented at the top page of Experimental Animal Division's Page (https://mus.brc.riken.jp: upper panel) and the search page (https://mus.brc.riken.jp/en/search_for_mouse_strain: lower panel). b An example of search result for mouse strains that have homologs related to the keyword entered are listed. c Detailed information page for a mouse strain including health reports, alleles and publications. The detailed page is linked to ordering information page for mouse strains

Genome Function. RIKEN BRC has committed to this effort. These collaborative activities will greatly contribute to delineate the biological gene function, a better understanding of diseases, drug discovery and development as well as prediction of potential side-effects early in the drug discovery process, and moreover, deeper insight into sophisticated biological functions. Phenotype data analyzed in RIKEN BRC by the comprehensive mouse phenotyping platform, Japan Mouse Clinic [33], is regularly transported to the data coordination center of IMPC through the data conversion pipeline which transforms raw experimental data to the standardized format in IMPC. Phenotype data analyzed in RIKEN BRC is available at the IMPC website (http://www. mousephenotype.org) with the result of automatic phenotype annotation by MP ontology. The IMPC dataset represents the largest and most reliable phenotype data using standardized genetic background, experimental methods [34] analysis workflows, and control strategies [35]. The mouse strains used in the phenotype analyses are available from RIKEN BRC.

To provide reliable, weighted phenotype-phenotype relationships as a reference data resource useful to detailed evaluation of the mouse mutant resources, RIKEN BRC worked out an association rule mining of a dataset consisting of only binary (normal and abnormal phenotypes) data to determine relationships among phenotypes with
IMPC data which is a bias-minimized comprehensive data of mouse phenotypes. As a result, a set of phenotypephenotype association pairs (PPAPs) as a module of phenotypic expression for each of the 345 phenotypes were defined [36, 37]. By analyzing each PPAP, phenotype sub-networks consisting of the phenotypes and distinct biological systems were also defined. Hierarchical clustering based on phenotype similarities among the 345 PPAPs revealed seven community types within a putative phenome-wide association network (Fig. 5). These mouse phenome-wide phenotype-phenotype association data reveal general principles of relationships among mammalian phenotypes and provide a reference resource for biomedical analyses. To promote use of the association data, we developed and published web-application tools available at: https://brc-riken.shinyapps.io/phenotypic_associations_ across_the_mouse_phenome/ and https://brc-riken.shinyapps.io/associations_between_biological_systems/.

\section{Future directions}

Above, we overviewed newly released information resources of mouse mutant strains in RIKEN BRC in 2020. Currently, mouse strains including GM mice are expected to contribute more and more as animal models of human diseases. Thus, the requirement for mouse information resources is becoming more sophisticated to 


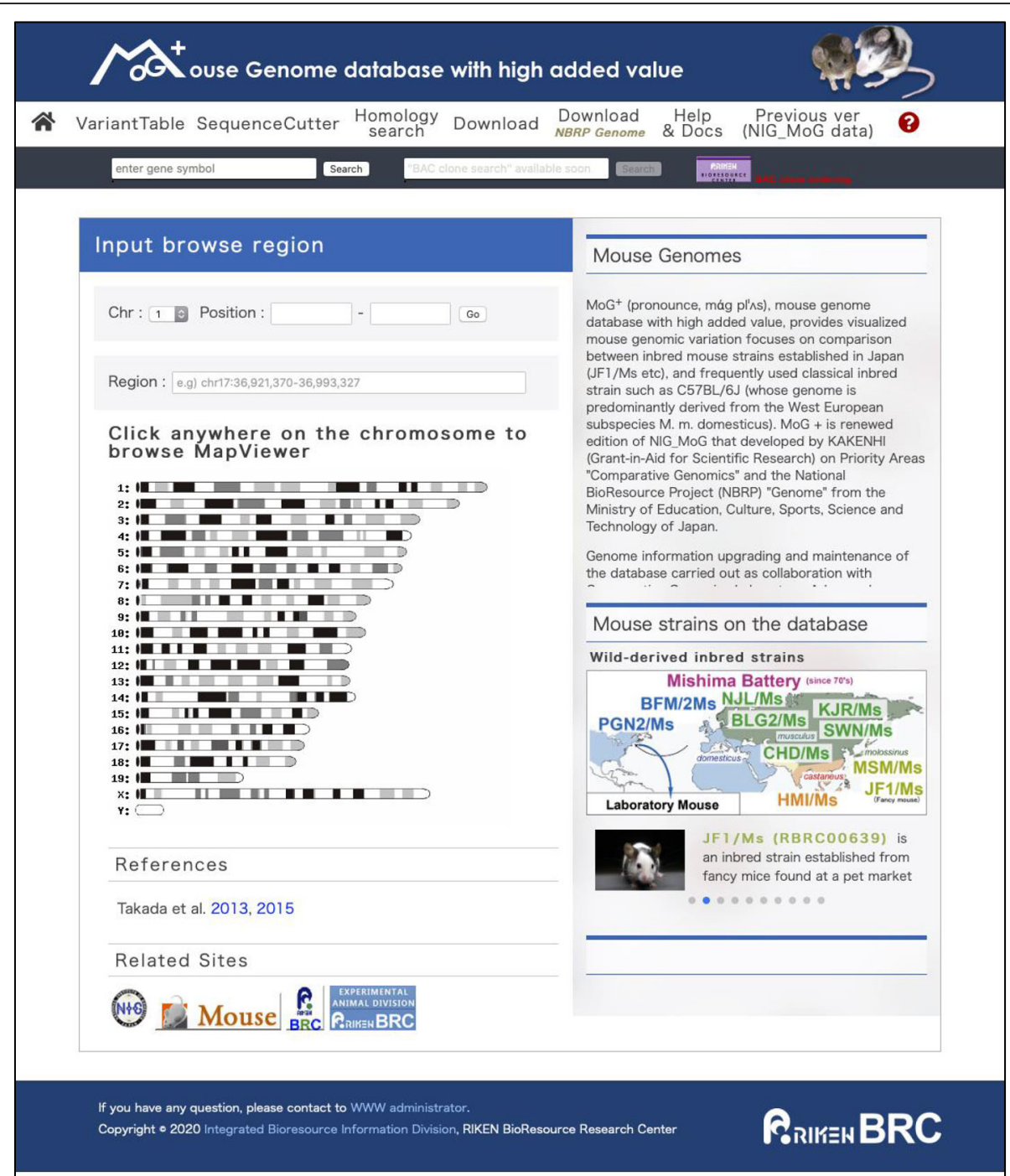

Fig. 4 The top page of MoG+ database (https://molossinus.brc.riken.jp). Detailed explanation of this web application is available at the tutorial page (https://github.com/ttakada1/MoGplus_tutorial_2020/wiki)

quickly respond to the needs of scientific communities. This trend has become more pronounced during the 2020 coronavirus crisis. Aiming to promote the advanced use and to help expand the use of mouse mutant resources, RIKEN BRC continuously supports the development of information technologies on data integration, implementation of advanced data searching system, and data analysis.

Further collaboration with RRID is a non-negligible issue for bioresource repositories. Although mouse strains and cultured cells in RIKEN BRC are already registered in the RRID database, we need to promote RRID for plant, microbes, and genetic material.

Further data integration across different datasets, online catalogs, genome variation databases and datasets produced from advanced mathematical methods such as the phenotype-phenotype association described above is also one of the major issues. One possible way to achieve this is to apply RDF-related technology to datasets such as $\mathrm{MoG}^{+}$and advanced data analysis methods and dataset derived from the data analysis. $\mathrm{MoG}^{+}$is planned to enhance through the collaboration with human genetic variation databases such as TogoVar (https://togovar. biosciencedbc.jp/) which collects and organizes human genomic variants in the Japanese population assorted by disease information [38] to provide mouse-human orthologous relationships of genetic variants which are valuable for modeling of human disease. Generation of an RDF version of variation data such as Variant Call Format (VCF) may be also valuable for global data integration of genetic variation and bioresource information. 


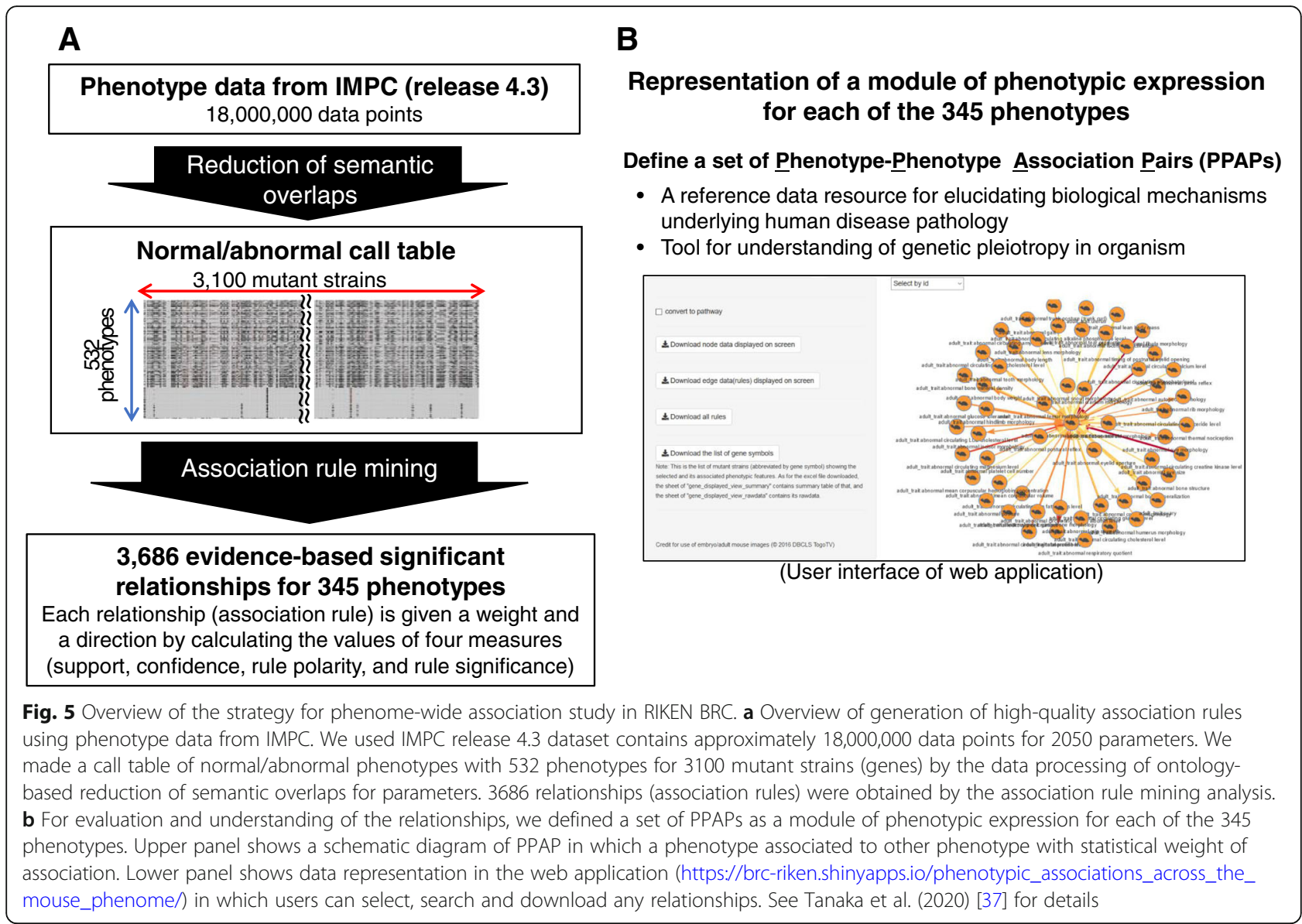

The online bioresource catalog in RIKEN BRC is planned to have enhanced search functions with better data integration with genomics, genetics, and phenotype data. The gene-based search of bioresources will be enhanced referring another dataset than Ensembl. We currently plan to introduce an RDF version of the Orthologous Matrix (OMA: https://omabrowser.org/ oma/home/) database [39] to our RDF store. The OMA database is a comprehensive resource to relate genes across a wide range of species generated by the OMA inference algorithm to identify orthologous pairs and hierarchical orthologous groups in which distinction between orthologs (genes or genomic segments in different species evolving from a common ancestral sequence) and paralogs (copies of genes or genomic segments created by a duplication events within the same species) is ensured. Improvement of the accuracy of gene search function to avoid listing too many genes with paralogous relationship is expected using OMA, in which orthologous and paralogous relationship are implemented as different relationships.

It is also planned the implementation of mouse strain search with vocabularies of phenotype and human disease. As described above, providing phenotype information is one of the most important functions required for the mouse information resource which is a serious weakness in the current version of RIKEN BRC mouse strain database. To address this, a curation of mouse strain phenotypes using MP ontology [12] is ongoing in RIKEN BRC. We also plan to introduce phenotype-related ontologies (MP, MA, HPO) which are available as Web Ontology Language (OWL: an RDFcompatible language for description of ontology) and data library to relate ontology terms of mouse phenotypes to human diseases [14-19] to our RDF store. In addition to these datasets, DisGenNET provides genedisease relationships [28]. Using these datasets in our RDF store or via the federated query function that allows data inquiry across different RDF stores, we plan to enhance the search function of the bioresource database to suggest mouse strains which are genetically or phenotypically related to human diseases. With these enhancements, RDF-based technology provides a cost-effective way of development and implementation.

Provision of advanced mathematical methods to analyze big-data is one of the most effective ways to add value to bioresources discovery of novel biological functions or principles of life systems. In addition to 
phenotype-phenotype associations, we also work in the development of a novel mathematical method termed "Energy landscape analysis" to visualize energy states or stabilities using omics data of microflora or transcriptome of environments or organisms [40]. This methodology is expected to be useful for controlling the biological state of soil, intestine, and cell differentiation which are composed of multiple factors with intricate interactions. Providing an RDF version of resultant data showing direct links to bioresources as materials of the experiment will significantly contribute to add value to bioresources. Sharing of workflow for computational data analysis using container virtualization technologies is another issue to consider [41].

There is a lot of information that should be shared to improve the value of bioresources. The Asian Mouse Mutagenesis Resource Association (AMMRA) is a collaborative group for the development, archiving/distribution, coordination of phenotyping, and informatics of mutant mice in Asia and Australia developed for sharing expertise, mouse modelling resources, and technology developments for the rapid assessment of variants through model-on-demand programs, biorepositories, and phenotyping clinics, to researchers and clinicians. At the AMMRA website (http://ammra.info/), we share information of mouse resources as well as the training of technicians, researchers, and clinicians in mouse genetics and resources. Further international collaboration in bioinformatics and data integration among repositories may greatly contribute to facilitate advanced infrastructure for life science.

\section{Conclusion}

The infrastructure of science to ensure reproducibility and application of data integration technology is nonnegligible for online data resources of mouse mutant repositories. In 2020, RIKEN BRC released three new databases of the integrated online catalog for bioresources including mouse strains, mouse genome variation database, and a database for phenotype-phenotype association. With the integration of other RDF resources such as Ensembl, the database of mouse strain provides better function of gene-based search. To ensure the addition of further value to the mouse strain resource as a common experimental material, further enhancement, integration, and collaboration of mouse information resources are planned to provide better use of the information with richness, organization and standardization.

\section{Abbreviation \\ SPARQL: A recursive acronym for SPARQL Protocol and RDF Query Language (https://en.wikipedia.org/wiki/SPARQL\#cite_note-2)}

\section{Acknowledgements}

We thank Mrs. Takatsuki T. and Dr. Kawashima S. in Database Center for Life Science and Dr. Kawamoto S. in National Institute of Genetics for discussion on data integration and standardization of bioresource data. We also thank members of Experimental Animal Division, Experimental Plant Division, Cell Engineering Division, Gene Engineering Division, Microbe Division and Integrated Bioresource Information Division in RIKEN BRC for the collaboration of data management and powerful discussions for writing of the manuscript. This work was supported by The Management Expenses Grant for RIKEN BioResource Research Center, MEXT, National BioResource Projects of "the Genome Information Upgrading Program" of AMED, MEXT Grant-in-Aid for Scientific Research on Innovative Areas FY2016-

2020Integrative Research toward Elucidation of Generative Brain Systems for Individuality, Cabinet Office, Government of Japan, Cross-ministerial Strategic Innovation Promotion Program (SIP), "Technologies for Smart Bio-industry and Agriculture"(funding agency: Bio-oriented Technology Research Advancement Institution, NARO)

\section{Authors' contributions}

Contributors HM was responsible for the overall development of databases in RIKEN BRC. DU, TK and NK were responsible for the development of the RDF-based integrate database of bioresources. YY and TK are responsible for data integration of bioresource and disease. NY, KK, YN and SI were responsible for the development of GUI of databases. $T T$ is responsible for the development of $\mathrm{MoG}^{+}$. NT is responsible for the development of weighted phenotype-phenotype relationship database. KS is responsible for data analyses and the energy landscape analysis. All authors contributed to the writing of the final manuscript. The author(s) read and approved the final manuscript.

\section{Funding}

This review referred the works funded by the Management Expenses Grant for RIKEN BioResource Research Center, MEXT (http://www.mext.go.jp/), Cross-ministerial Strategic Innovation Promotion Program (SIP), "Technologies for Smart Bio-Industry and Agriculture" and JSPS KAKENHI Grant Numbers: JP18H03298 and JP16K14600. Genome information analysis for MoG+ was supported by National Institute of Genetics Advanced Genomics Project (Next Generation Genome-Research-Hub Project), "Genome Information Upgrading Program" of National BioResource Project (NBRP) from Japan Agency for Medical Research and Development (AMED), Grant-in-Aid for Scientific Research on Priority Areas in "Comparative Genomics", and also supported in part by the Biodiversity Research Project of the Transdisciplinary Research Integration Center, Research Organization of Information and Systems.

\section{Availability of data and materials}

Datasets described in this review are available at https://knowledge.brc.riken. jp, https://molossinus.brc.riken.jp/pub/, https://brc-riken.shinyapps.io/ phenotypic_associations_across_the_mouse_phenome/ and https://brcriken.shinyapps.io/associations_between_biological_systems/

\section{Competing interests}

The authors have no competition of interest directly relevant to the content of this article.

\section{Author details \\ ${ }^{1}$ Integrated Bioresource Information Division, RIKEN BioResource Research Center, 3-1-1 Koyadai, Tsukuba-shi, Ibaraki 305-0074, Japan. ${ }^{2}$ Experimental Animal Division, BioResource Research Center, RIKEN, Tsukuba, Japan. ${ }^{3}$ Laboratory for Developmental Dynamics, Center for Biosystems Dynamics Research, RIKEN, Kobe, Japan. ${ }^{4}$ Data Knowledge Organization Unit, Head Office for Information Systems and Cybersecurity, RIKEN, Wako, Japan.}

Received: 10 July 2020 Accepted: 21 September 2020

Published online: 18 January 2021

\section{References}

1. Eppig JT, Richardson JE, Kadin JA, Ringwald M, Blake JA, Bult CJ. Mouse genome informatics (MGl): reflecting on 25 years. Mamm Genome. 2015;26: 272-84. https://doi.org/10.1007/s00335-015-9589-4.

2. Law M, Shaw DR. Mouse genome informatics (MGI) is the international resource for information on the laboratory mouse. Methods Mol Biol. 1757; 2018:141-61. https://doi.org/10.1007/978-1-4939-7737-6_7. 
3. Maltais LJ, Blake JA, Eppig JT, Davisson MT. Rules and guidelines for mouse gene nomenclature: a condensed version. International committee on standardized genetic nomenclature for mice. Genomics. 1997;45:471-6.

4. Wain HM, Lush M, Ducluzeau F, Povey S. Genew: the human gene nomenclature database. Nucleic Acids Res. 2002;30:169-71.

5. Twigger SN, Shimoyama M, Bromberg S, Kwitek AE, Jacob HJ, RGD team. The rat genome database, update 2007--easing the path from disease to data and back again. Nucleic Acids Res. 2007;35:D658-62.

6. Sayers EW, Barrett T, Benson DA, Bryant SH, Canese K, Chetvernin V, Church DM, DiCuccio M, Edgar R, Federhen S, et al. Database resources of the National Center for biotechnology information. Nucleic Acids Res. 2009;37:D5-15.

7. Hubbard TJ, Aken BL, Ayling S, Ballester B, Beal K, Bragin E, Brent S, Chen Y, Clapham P, Clarke L, et al. Ensembl 2009. Nucleic Acids Res. 2009;37:D690-7.

8. Eppig JT, Motenko H, Richardson JE, Richards-Smith B, Smith CL. The international mouse strain resource (IMSR): cataloging worldwide mouseand ES cell line resources. Mamm Genome. 2015;26:448-55. https://doi.org/10. 1007/s00335-015-9600-0.

9. Smith B, Ashburner M, Rosse C, Bard J, Bug W, Ceusters W, Goldberg LJ, Eilbeck K, Ireland A, Mungall CJ, et al. The OBO foundry: coordinated evolution of ontologies to support biomedical data integration. Nat Biotechnol. 2007;25:1251-5.

10. Ashburner, et al. Gene ontology: tool for the unification of biology. Nat Genet. 2000;25:25-9.

11. Hayamizu TF, Baldock RA, Ringwald M. Mouse anatomy ontologies: enhancements and tools for exploring and integrating biomedical data. Mamm Genome. 2015;26(9-10):422-30. https://doi.org/10.1007/s00335015-9584-9.

12. Smith CL, Eppig JT. The mammalian phenotype ontology: enabling robust annotation and comparative analysis. Wiley Interdiscip Rev Syst Biol Med. 2009;1:390-9. https://doi.org/10.1002/wsbm.44.

13. Mallon A-M, lyer $V$, Melvin D, Morgan $H$, Parkinson $H$, Brown SDM, Flicek $P$, William C. Skarnes. Accessing data from the International Mouse Phenotyping Consortium: state of the art and future plans. Mamm Genome. 2012;23:641-52. https://doi.org/10.1007/s00335-012-9428-9.

14. Robinson PN, Mundlos S. The human phenotype ontology. Clin Genet. 2010;77(6):525-34. https://doi.org/10.1111/j.1399-0004.2010.01436.x Epub 2010 Feb 11. PMID: 20412080 Review.

15. Washington NL, Haendel MA, Mungall CJ, Ashburner M, Westerfield M, Lewis SE. Linking human diseases to animal models using ontology-based phenotype annotation. PLoS Biol. 2009;7:e1000247. https://doi.org/10.1371/ journal.pbio.1000247.

16. Hoehndorf R, Schofield PN, Gkoutos GV. PhenomeNET: a whole-phenome approach to disease gene discovery. Nucleic Acids Res. 2011;39:e119. https://doi.org/10.1093/nar/gkr538.

17. Mungall CJ, Torniai C, Gkoutos GV, Lewis SE, Haendel MA. Uberon, an integrative multi-species anatomy ontology. Genome Biol. 2012;13:R5. https://doi.org/10.1186/gb-2012-13-1-r5.

18. Köhler S, Doelken SC, Ruef BJ, Bauer S, Washington N, Westerfield M, Gkoutos G, Schofield P, Smedley D, Lewis SE, Robinson PN, Mungall CJ. Construction and accessibility of a cross-species phenotype ontology along with gene annotations for biomedical research. F1000Res. 2013;2:30. https:// doi.org/10.12688/f1000research.2-30.v2.

19. Smedley D, Oellrich A, Köhler S. Ruef B; sanger mouse genetics project, Westerfield M, Robinson P, Lewis S, Mungall C. PhenoDigm: analyzing curated annotations to associate animal models with human diseases. Database (Oxford). https://doi.org/10.1093/database/bat025.

20. Bandrowski A, Brush M, Grethe JS, Haendel MA, Kennedy DN, Hill S, Hof PR, Martone ME, Pols M, Tan SS, Washington N, Zudilova-Seinstra E. Vasilevsky $\mathrm{N}$; RINL resource identification initiative. The resource identification initiative: A cultural shift in publishing. Neuroinformatics. 2016;14:169-82. https://doi. org/10.1007/s12021-015-9284-3.

21. Berners-Lee T, Hendler J, Lassila O. The Semantic Web. Sci Am. 2001;284:3443.

22. World Wide Web Consortium. Resource Description Framework (RDF) Model and Syntax Specification W3C Proposed Recommendation 05 January 1999. 1999. https://www.w3.org/TR/PR-rdf-syntax/Overview.html Accessed 29 May 2020

23. Wilkinson MD, Dumontier M, Aalbersberg IJ, Appleton G, Axton M, Baak A, Blomberg N, Boiten JW, da Silva Santos LB, Bourne PE, Bouwman J, Brookes AJ, Clark T, Crosas M, Dillo I, Dumon O, Edmunds S, Evelo CT, Finkers R,
Gonzalez-Beltran A, Gray AJ, Groth P, Goble C, Grethe JS, Heringa J, 't Hoen PA, Hooft R, Kuhn T, Kok R, Kok J, Lusher SJ, Martone ME, Mons A, Packer AL, Persson B, Rocca-Serra P, Roos M, van Schaik R, Sansone SA, Schultes E, Sengstag T, Slater T, Strawn G, Swertz MA, Thompson M, van der Lei J, van Mulligen E, Velterop J, Waagmeester A, Wittenburg P, Wolstencroft K, Zhao J, Mons B. The FAIR Guiding Principles for scientific data management and stewardship. Sci Data. 2016; 15;3:160018. doi: https:// doi.org/10.1038/sdata.2016.18.

24. Yoshiki A, Ike F, Mekada K, Kitaura Y, Nakata H, Hiraiwa N, Mochida K, ljuin M, Kadota M, Murakami A, Ogura A, Abe K, Moriwaki K, Obata Y. The mouse resources at the RIKEN BioResource center. Exp Anim. 2009;58:85-96.

25. Masuya H, Makita Y, Kobayashi N, Nishikata K, Yoshida Y, Mochizuki Y, Doi K, Takatsuki T, Waki K, Tanaka N, Ishii M, Matsushima A, Takahashi S, Hijikata A, Kozaki K, Furuichi T, Kawaji H, Wakana S, Nakamura Y, Yoshiki A, Murata T, Fukami-Kobayashi K, Mohan S, Ohara O, Hayashizaki Y, Mizoguchi R, Obata Y, Toyoda T. The RIKEN integrated database of mammals. Nuc leic Acids Res. 2011;39:D861-70. https://doi.org/10.1093/nar/gkq1078.

26. Kobayashi N, Kume S, Lenz K, Masuya H. RIKEN MetaDatabase: A Database Platform for Health Care and Life Sciences as a Microcosm of Linked Open Data Cloud. Int J Semant Web Inf Syst. 2018;14:140-64.

27. Jupp S, Malone J, Bolleman J, Brandizi M, Davies M, Garcia L, Gaulton A, Gehant S, Laibe C, Redaschi N, Wimalaratne SM, Martin M, Le Novère N, Parkinson $\mathrm{H}$, Birney $\mathrm{E}$, Jenkinson AM. The EBI RDF platform: linked open data for the life sciences. Bioinformatics. 2014;30:1338-9. https://doi.org/10. 1093/bioinformatics/btt765 Epub 2014 Jan 11.

28. Piñero J, Ramírez-Anguita JM, Saüch-Pitarch J, Ronzano F, Centeno E, Sanz F, Furlong LI. The DisGeNET knowledge platform for disease genomics: 2019 update. Nucleic Acids Res. 2020;48(D1):D845-55. https://doi.org/10.1093/nar/ gkz1021 PMID: 31680165.

29. Saul MC, Philip VM, Reinholdt LG, Center for Systems Neurogenetics of addiction, Chesler EJ. High-diversity mouse populations for complex traits. Trends Genet. 2019:35:501-14. https://doi.org/10.1016/j.tig.2019.04.003 Epub 2019 May 24.

30. Takada T, Ebata T, Noguchi H, Keane TM, Adams DJ, Narita T, Shin-I T, Fujisawa H, Toyoda A, Abe K, Obata Y, Sakaki Y, Moriwaki K, Fujiyama A, Kohara $Y$, Shiroishi $T$. The ancestor of extant Japanese fancy mice contributed to the mosaic genomes of classical inbred strains. Genome Res. 2013;23(8):1329-38. https://doi.org/10.1101/gr.156497.113 Epub 2013 Apr 19

31. Takada T, Yoshiki A, Obata Y, Yamazaki Y, Shiroishi T. NIG_MoG: a mouse genome navigator for exploring intersubspecific genetic polymorphisms. Mamm Genome. 2015;26(7-8):331-7. https://doi.org/10.1007/s00335-015-9569-8.

32. Brown SDM, Holmes CC, Mallon AM, Meehan TF, Smedley D, Wells S. Highthroughput mouse phenomics for characterizing mammalian gene function. Nat Rev Genet. 2018;19:357-70. https://doi.org/10.1038/s41576-018-0005-2.

33. Wakana S, Suzuki T, Furuse T, Kobayashi K, Miura I, Kaneda H, Yamada I, Motegi H, Toki H, Inoue M, Minowa O, Noda T, Waki K, Tanaka N, Masuya H, Obata Y. Introduction to the Japan mouse clinic at the RIKEN BioResource center. Exp Anim. 2009;58:443-50. https://doi.org/10.1538/expanim.58.443.

34. Mandillo S, Tucci V, Hölter SM, Meziane H, Banchaabouchi MA, Kallnik M, Lad HV, Nolan PM, Ouagazzal AM, Coghill EL, Gale K, Golini E, Jacquot S, Krezel W, Parker A, Riet F, Schneider I, Marazziti D, Auwerx J, Brown SD, Chambon P, Rosenthal N, Tocchini-Valentini G, Wurst W. Reliability, robustness, and reproducibility in mouse behavioral phenotyping: a crosslaboratory study. Physiol Genomics. 2008;34:243-55. https://doi.org/10.1152/ physiolgenomics.90207.

35. Karp NA, Speak AO, White JK, et al. Impact of temporal variation on design and analysis of mouse knockout phenotyping studies. PLoS One. 2014;9: e111239. https://doi.org/10.1371/journal.pone.0111239.

36. Tanaka N, Masuya $H_{\text {., }}$ Mouse phenome as a biological resource., Impact. 2018; 12: 93-95. DOl: https://doi.org/10.21820/23987073.2018.12.93.

37. Tanaka N, Masuya H. An atlas of evidence-based phenotypic associations across the mouse phenome. Sci Rep. 2020;10:3957 https://www.nature.com/ articles/s41598-020-60891-w.

38. Japan Science and Technology Agency, JST releases 'TogoVar', an integrated database for Japanese genome variants/variations, EurekAlert Public Release. 2018; 10-JUL-2018 (https://www.eurekalert.org/pub_ releases/2018-07/jsat-jr071018.php).

39. Altenhoff AM, Glover NM, Train CM, et al. The OMA orthology database in 2018: retrieving evolutionary relationships among all domains of life through richer web and programmatic interfaces. Nucleic Acids Res. 2018; 46(D1):D477-85. https://doi.org/10.1093/nar/gkx1019. 
40. Suzuki K, Nagaoka S, Fukuda S and Masuya H, Energy landscape analysis of ecological communities elucidates the phase space of community assembly dynamics. Ecological Monographs 2020; in review.

41. Ohta T, Tanjo T, Ogasawara O. Accumulating computational resource usage of genomic data analysis workflow to optimize cloud computing instance selection. Gigascience. 2019;8:giz052. https://doi.org/10.1093/gigascience/giz052.

\section{Publisher's Note}

Springer Nature remains neutral with regard to jurisdictional claims in published maps and institutional affiliations.

Ready to submit your research? Choose BMC and benefit from:

- fast, convenient online submission

- thorough peer review by experienced researchers in your field

- rapid publication on acceptance

- support for research data, including large and complex data types

- gold Open Access which fosters wider collaboration and increased citations

- maximum visibility for your research: over $100 \mathrm{M}$ website views per year

At BMC, research is always in progress.

Learn more biomedcentral.com/submissions 